\title{
Type VII Collagen: From Discovery of the Anchoring Fibril Protein to Clinical Trials Ameliorating the Human Blistering Disease Epidermolysis Bullosa
}

\author{
Douglas R. Keene ${ }^{1}$ and Sara F. Tufa ${ }^{1}$ \\ ${ }^{1}$ Shriners Hospital for Children, Micro-Imaging Center, Portland, Oregon USA
}

Type VII collagen (C7) was identified in 1983, initially as a novel band on an electrophoretic gel separating the components of a tissue homogenate [1]. Joining this group in 1986, our laboratory utilized rotary shadowing to characterize type VII collagen as a dimeric molecule, approximately 790 $\mathrm{nm}$ in length, composed of two anti-parallel monomers (inset, Figure 1A) overlapping at their NC2terminal ends by 60nm. Using antibodies prepared in mice, we identified C7 as a component of anchoring fibrils. Anchoring fibrils are long banded structures anchored at both ends within the lamina densa of the dermal-epidermal junction. At the middle of the molecule, arches form within the shallow papillary dermis (Figure 1A). The resulting loops entrap dermal collagen fibrils, forming "staples" which mechanically fasten the epithelium to the underlying dermis.

Recognizing that a defect in this adhesive molecule might result in a subepidermal split causing blistering, candidate diseases with blistering phenotypes were evaluated. Recessive Epidermolysis Bullosa (RDEB) results in severe blistering, mutilating scarring, and predilection for squamous cell carcinoma. Mutations were found in the COL7A1 gene resulting in diminished or absent C7 and consequential lack of functional anchoring fibrils (Figures 1B, C).

We then evaluated therapies to deliver functional C7 to the basement membrane of C7 deficient skin. Our collaborators at Stanford, led by Paul Khavari, Peter Marinkovich and Alfred Lane, regenerated epidermis from retrovirally transduced autologous RDEB keratinocytes, allowing long-term expression of C7 in human EB skin transplanted onto immunodeficient mice [2]. Meanwhile, our collaborators in Minnesota, led by Jakub Tolar, demonstrated that transplantation of normal bone marrow into C7 deficient EB mice was effective in ameliorating the disease phenotype, with the production of functional C7 and anchoring fibrils at the DEJ [3].

Having tested these two therapies in mice, clinical trials to ameliorate the disease in humans began. Jakub Tolar's group focuses on using bone marrow transplant therapy to deliver WT stem cells to the skin. These cells appear to enter the skin via the circulatory system; they then incorporate into the epithelium and produce functional type VII collagen. Patients are biopsied at 0, 30, 60, 90,180 days then yearly post-transplant and evaluated by ILM and ITEM. Some patients respond to therapy with the production of near normal density of anchoring fibrils (Figure 2) but in a significant number of individuals the anchoring fibril architecture is often thin and wispy, forming only occasional fibrils that are non-banded and mostly without loops. Still, blistering is markedly improved and patient quality of life is decidedly enriched, with significant decrease in blister formation [4]. These individuals give credibility to the suggestion that only $30 \%$ of normal C7 is required for skin integrity.

The clinical trial at Stanford seeks to treat highly involved lesions by transplanting corrected patient keratinocyte skin grafts onto prepared would beds (Figure 3). Patients are evaluated by ILM and ITEM at day 0, then within 30 days and again at 3,6 and 12 months post grafting. Complete epidermal regeneration has been observed within 30days. Immunocytochemistry reveals strong C7 expression at 
the DEJ and a robust anchoring fibril network. Six transplants have been done in each of four patients to date, with encouraging outcomes in all.
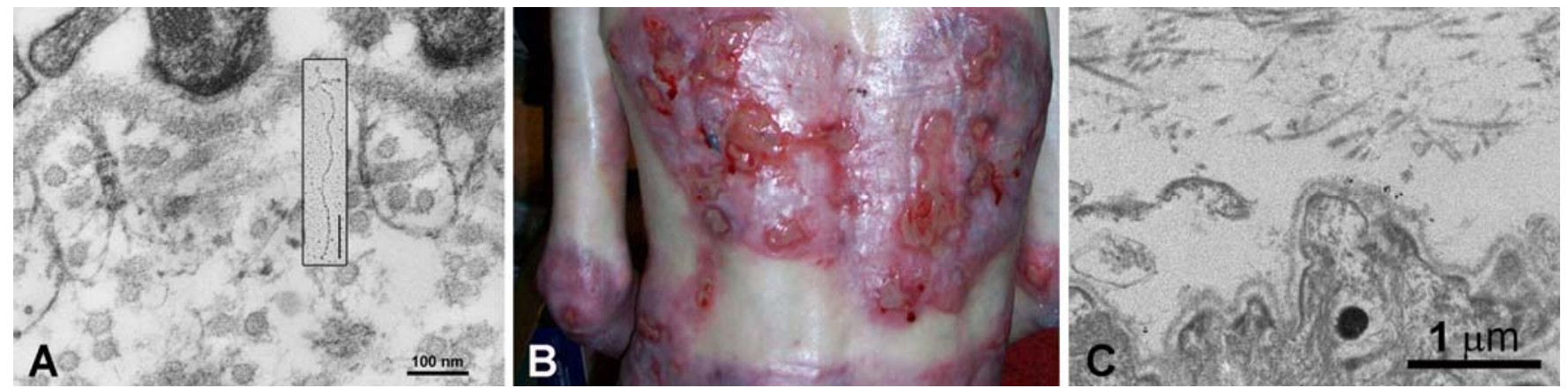

Figure 1. Anchoring fibrils (formed by C7, rotary shadow inset) entrap banded collagen to "staple" epithelium to dermis in normal skin (A). The severe blistering of Recessive Dystrophic Epidermolysis Bullosa (B) results from a lack of functional C7 and subsequent lack of anchoring fibrils (C).

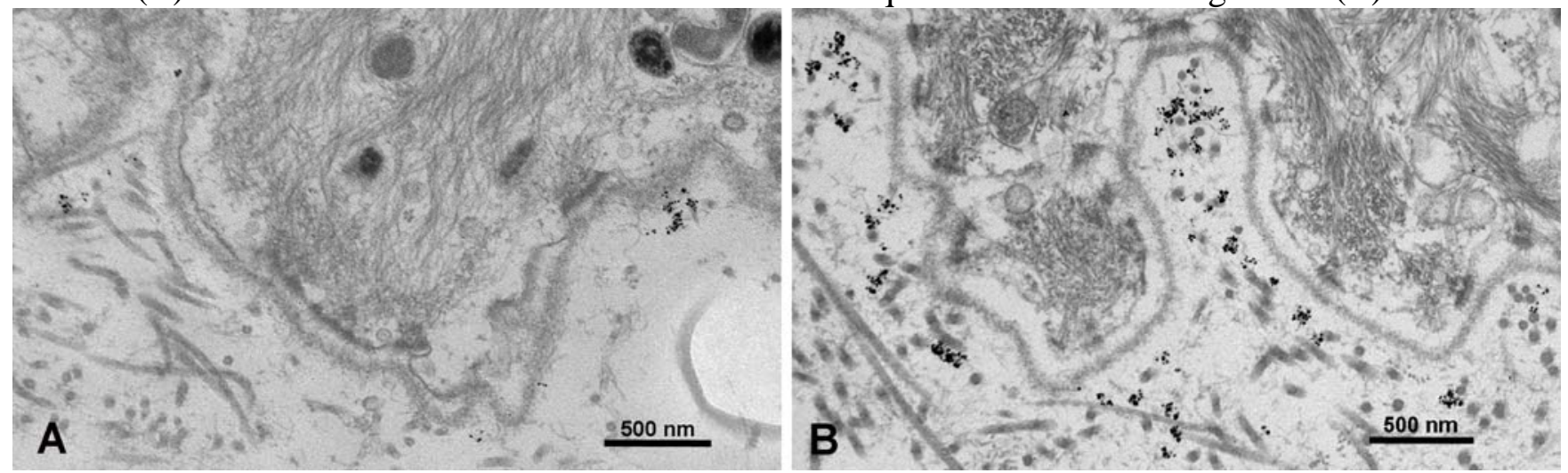

Figure 2. Anchoring fibril immuno-density at day 0 is nil (A) but at day 360 post bone marrow transplant this patient has near normal immuno-density and ultrastructurally identifiable AFs (B).
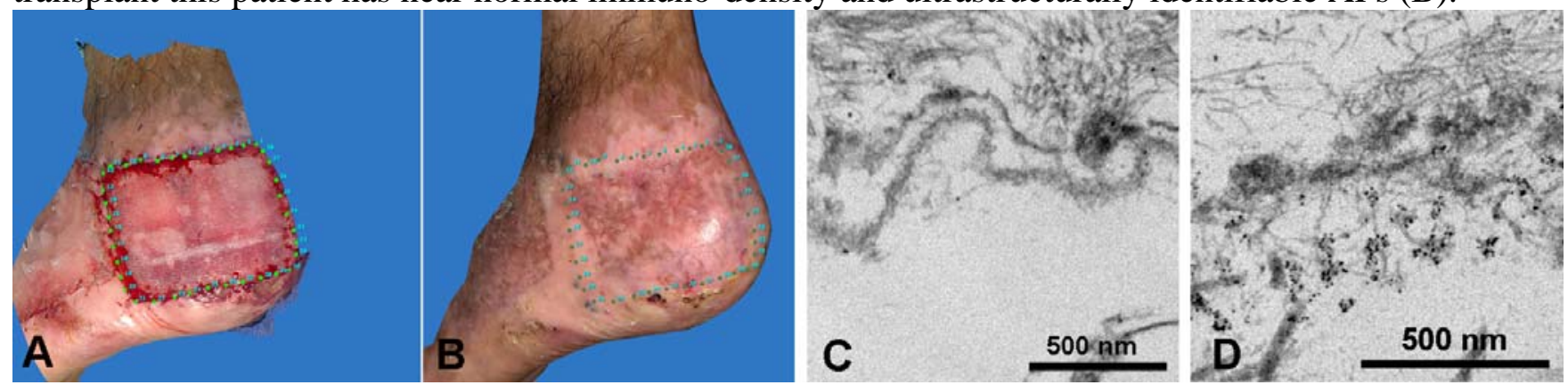

Figure 3. A region of severe blistering has been prepared and grafted (day 0, A, C). After 180 days the grafted region lacks blisters and has gained a population of immuno-identifiable anchoring fibrils (B,D)

\section{References:}

[1] H Bentz et al. (1983). Proc Natl Acad Sci U S A. 80:3168-72.

[2] S Ortiz-Urda1 et al. (2002). Nature Medicine 8, 1166 - 1170.

[3] J Tolar et al. (2009). Blood. 113:1167-74.

[4] J E Wagner et al. (2010). N Engl J Med. 363:629-39.

[5] Funding for this work was generously provided by the Shriners Hospitals for Children 